\title{
Optimal Control Problem for Switched System with the Nonsmooth Cost Functional
}

\author{
Shahlar Meherrem ${ }^{1}$ and Rufan Akbarov ${ }^{2}$ \\ ${ }^{1}$ Department of Mathematics, Yasar University, 35100 Izmir, Turkey \\ 2 Department of Business Administration, Cologne University, 50931 Cologne, Germany
}

Correspondence should be addressed to Shahlar Meherrem; magerramovsf@hotmail.com

Received 2 July 2013; Revised 15 August 2013; Accepted 3 September 2013

Academic Editor: Nazim Idrisoglu Mahmudov

Copyright (C) 2013 S. Meherrem and R. Akbarov. This is an open access article distributed under the Creative Commons Attribution License, which permits unrestricted use, distribution, and reproduction in any medium, provided the original work is properly cited.

\begin{abstract}
We examine the relationships between lower exhausters, quasidifferentiability (in the Demyanov and Rubinov sense), and optimal control for switching systems. Firstly, we get necessary optimality condition for the optimal control problem for switching system in terms of lower exhausters. Then, by using relationships between lower exhausters and quasidifferentiability, we obtain necessary optimality condition in the case that the minimization functional satisfies quasidifferentiability condition.
\end{abstract}

\section{Introduction}

A switched system is a particular kind of hybrid system that consists of several subsystems and a switching law specifying the active subsystem at each time instant. There are some articles which are dedicated to switching system [1-8]. Examples of switched systems can be found in chemical processes, automotive systems, and electrical circuit systems, and so forth.

Regarding the necessary optimality conditions for switching system in the smooth cost functional, it can be found in $[1,4,6]$. The more information connection between quasidifferential, exhausters and Hadamard differential are in [8-10]. Concerning the necessary optimality conditions for discrete switching system is in [5], and switching system with Frechet subdifferentiable cost functional is in [3]. This paper addresses the role exhausters and quasi-differentiability in the switching control problem. This paper is also extension of the results in the paper [5] (additional conditions are switching points unknown, and minimization functional is nonsmooth) in the case of first optimality condition. The rest of this paper is organized as follows. Section 2 contains some preliminaries, definitions, and theorems. Section 3 contains problem formulations and necessary optimality conditions for switching optimal control problem in the terms of exhausters. Then, the main theorem in Section 3 is extended to the case in which minimizing function is quasidifferentiable.

\section{Some Preliminaries of Non-Smooth Analysis}

Let us begin with basic constructions of the directional derivative (or its generalization) used in the sequel. Let $f$ : $X \rightarrow R, X \subset R^{n}$ be an open set. The function $f$ is called Hadamard upper (lower) derivative of the function $f$ at the point $x \in X$ in the direction $g \in X$ if there exist limit such that

$$
\begin{gathered}
f_{H}^{\uparrow}:=\lim _{[\alpha, g] \rightarrow[+0, g]} \frac{1}{\alpha}[f(x+\alpha g)-f(x)], \\
\left(f_{H}^{\downarrow}:=\liminf _{[\alpha, g] \rightarrow[+0, g]} \frac{1}{\alpha}[f(x+\alpha g)-f(x)]\right),
\end{gathered}
$$

where $[\alpha, g] \rightarrow[+0, g]$ means that $\alpha \rightarrow+0$ and $g \rightarrow g$.

Note that limits in (1) always exist, but there are not necessary finite. This derivative is positively homogeneous functions of direction. The Gateaux upper (lower) subdifferential of the function $f$ at a point $x_{0} \in X$ can be defined as follows:

$$
\begin{gathered}
\partial_{G}^{+} f\left(x_{0}\right)=\left\{v \in R^{n} \mid \limsup _{t \downarrow 0} \frac{f\left(x_{0}+t g\right)-f\left(x_{0}\right)}{t}\right. \\
\left.\leq(v, g), \quad \forall g \in R^{n}\right\} .
\end{gathered}
$$


The set

$$
\widehat{\partial}^{+} f\left(x_{0}\right)=\left\{v \in R^{n} \mid \limsup _{x \rightarrow x_{0}} \frac{f(x)-f\left(x_{0}\right)-\left\langle v, x-x_{0}\right\rangle}{\left\|x-x_{0}\right\|} \leq 0\right\}
$$

is called, respectively, the upper (lower) Frechet subdifferential of the function $f$ at the point $x_{0}$.

As observed in $[9,10]$, if $f$ is a quasidifferentiable function then its directional derivative at a point $x$ is represented as

$$
f^{\prime}(x, g)=\max _{v \in \underline{\partial} f(x)}(v, g)+\min _{w \in \bar{\partial} f(x)}(w, g),
$$

where $\underline{\partial} f(x), \bar{\partial} f(x) \subset R^{n}$ are convex compact sets. From the last relation, we can easily reduce that

$$
f^{\prime}(x, g)=\min _{w \in \bar{\partial} f(x)} \max _{v \in w+\underline{\partial} f(x)}(v, g)=\max _{v \in \underline{\partial} f(x)} \max _{w \in v+\bar{\partial} f(x)}(v, g) .
$$

This means that for the function $h(g)=f^{\prime}(x, g)$ the upper and lower exhausters can be described in the following way:

$$
\begin{aligned}
& E^{*}=\{C=w+\underline{\partial} f(x) \mid w \in \bar{\partial} f(x)\}, \\
& E_{*}=\{C=v+\bar{\partial} f(x) \mid v \in \underline{\partial} f(x)\} .
\end{aligned}
$$

It is clear that the Frechet upper subdifferential can be expressed with the Hadamard upper derivative in the following way; see [9, Lemma 3.2]:

$$
\partial_{F}^{+} f\left(x_{0}\right)=\partial_{F}^{+} f_{H}^{\uparrow}\left(x_{0}, 0_{n}\right) .
$$

Theorem 1. Let $E_{*}$ be lower exhausters of the positively homogeneous function $h: R^{n} \rightarrow R$. Then, $\bigcap_{C \subset E_{*}} C=\widehat{\partial}^{+} h\left(0_{n}\right)$, where $\widehat{\partial}^{+} h$ is the Frechet upper subdifferential of the $h$ at $0_{n}$, and for the positively homogeneous function $h: R^{n} \rightarrow R$ the Frechet superdifferential at the point zero follows

$$
\widehat{\partial}^{+} h\left(0_{n}\right)=\left\{v \in R^{n} \mid h(x)-(v, x) \leq 0, x \in R^{n}\right\} .
$$

Proof. Take any $v_{0} \in \bigcap_{C \subset E_{*}} C$. Then by using definition an lower exhausters we can write

$$
v_{0}(x) \geq h(x), \quad \forall x \in R^{n} \Longrightarrow \bigcap_{C \subset E_{*}} C \subset \widehat{\partial}^{+} h\left(0_{n}\right) .
$$

Consider now any $v_{0} \in \widehat{\partial}^{+} h\left(0_{n}\right) \Rightarrow$

$$
v_{0}(x) \geq h(x) .
$$

Let us consider $v_{0} \notin \bigcap_{C \subset E_{*}} C$. Then, there exists $C_{0} \in E^{*}$ where $v_{0} \notin C_{0}$. Then, by separation theorem, there exists $x_{0} \in$ $R^{n}$ such that

$$
\left(x_{0}, v_{0}\right) \leq \max _{v \in C_{0}}\left(x_{0}, v\right) \leq h(x) .
$$

It is conducts (3) and $v_{0} \in C$ for every $C \in E^{*}$ and due to arbitrary. This means that $v_{0} \in \bigcap_{C \subset E_{*}} C$. The proof of the theorem is complete.
Lemma 2. The Frechet upper and Gateaux lower subdifferentials of a positively homogeneous function at zero coincide.

Proof. Let $h: R^{n} \rightarrow R$ be a positively homogenous function. It is not difficult to observe that every $g \in R^{n}$ and every $t>0$ :

$$
\frac{h\left(0_{n}+t g\right)-h\left(0_{n}\right)}{t}=\frac{t h(g)}{t}=h(g) .
$$

Hence, the Gateaux lower subdifferential of $h$ at $0_{n}$ takes the forms

$$
\partial_{G}^{+} h\left(0_{n}\right)=\left\{v \in R^{n} \mid h(g) \leq(v, g), \forall g \in R^{n}\right\}
$$

which coincides with the representation of the Frechet upper subdifferential of the positively homogenous function (see [11, Proposition 1.9]).

\section{Problem Formulation and Necessary Optimality Condition}

Let investigating object be described by the differential equation

$$
\begin{array}{r}
\dot{x}_{K}(t)=f_{K}\left(x_{K}(t), u_{K}(t), t\right), \quad t \in\left[t_{k-1}, t_{k}\right], \\
K=1,2, \ldots, N
\end{array}
$$

with initial condition

$$
x_{1}\left(t_{0}\right)=x_{0}
$$

and the phase constraints at the end of the interval

$$
F_{K}\left(x_{N}\left(t_{N}\right), t_{N}\right)=0, \quad K=1,2, \ldots, N
$$

and switching conditions on switching points (the conditions which determine that at the switching points the phase trajectories must be connected to each other by some relations):

$$
x_{K+1}\left(t_{K}\right)=M_{K}\left(x_{K}\left(t_{K}\right), t_{k}\right), \quad K=1,2, \ldots, N-1 .
$$

The goal of this paper is to minimize the following functional:

$$
\begin{aligned}
S\left(u_{1}, \ldots, u_{N}, t_{1}, \ldots, t_{N}\right)= & \sum_{K=1}^{N} \varphi_{K}\left(x_{K}\left(t_{K}\right)\right) \\
& +\sum_{K=1}^{N} \int_{t_{K-1}}^{t_{K}} L\left(x_{K}(t), u_{K}(t), t\right) d t
\end{aligned}
$$

with the conditions (14)-(16). Namely, it is required to find the controls $u_{1}, u_{2}, \ldots, u_{N}$, switching points $t_{1}, t_{2}, \ldots, t_{N-1}$, and the end point $t_{N}$ (here $t_{1}, t_{2}, \ldots, t_{N}$ are not fixed) with the corresponding state $x_{1}, x_{2}, \ldots, x_{N}$ satisfying (14)-(16) so that the functional $J\left(u_{1}, \ldots, u_{N}, t_{1}, \ldots, t_{N}\right)$ in (18) is minimized. We will derive necessary conditions for the nonsmooth version of these problems (by using the Frechet superdifferential and exhausters, quasidifferentiable in the Demyanov and Rubinov sense). 
Here $f_{K}: \mathbb{R} \times \mathbb{R}^{n} \times \mathbb{R}^{r} \rightarrow \mathbb{R}^{n}, M_{K}$ and $F_{K}$ are continuous, at least continuously partially differentiable vectorvalued functions with respect to their variables, $L: \mathbb{R}^{n} \times \mathbb{R}^{r} \times$ $\mathbb{R} \rightarrow \mathbb{R}$ are continuous and have continuous partial derivative with respect to their variables, $\varphi_{k}(\cdot)$ has Frechet upper subdifferentiable (superdifferentiable) at a point $\bar{x}_{K}\left(t_{K}\right)$ and positively homogeneous functional, and $u_{K}(t): \mathbb{R} \rightarrow$ $U_{K} \subset \mathbb{R}^{r}$ are controls. The sets $U_{K}$ are assumed to be nonempty and open. Here (16) is switching conditions. If we denote this as follows: $\theta=\left(t_{1}, t_{2}, \ldots, t_{N}\right), x(t)=\left(x_{1}(t)\right.$, $\left.x_{2}(t), \ldots, x_{N}(t)\right), u(t)=\left(u_{1}(t), u_{2}(t), \ldots, u_{N}(t)\right)$, then it is convenient to say that the aim of this paper is to find the triple $(x(t), u(t), \theta)$ which solves problem (14)-(18). This triple will be called optimal control for the problem (14)-(18). At first we assume that $\varphi_{k}(\cdot)$ is the Hadamar upper differentiable at the point $\bar{x}_{K}\left(t_{K}\right)$ in the direction of zero. Then, $\varphi_{k}(\cdot)$ is upper semicontinuous, and it has an exhaustive family of lower concave approximations of $\varphi_{k}(\cdot)$.

Theorem 3 (Necessary optimality condition in terms of lower exhauster). Let $\left(\bar{u}_{K}(\cdot), \bar{x}_{K}(\cdot), \bar{\theta}\right)$ be an optimal solution to the control problem (14)-(18). Then, for every element $x_{K}^{*}$ from intersection of the subsets $C_{K}$ of the lower exhauster $E_{*, K}$ of the functional $\varphi_{K}\left(\bar{x}_{K}\left(\bar{t}_{K}\right)\right)$, that is, $x_{K}^{*} \in \bigcap_{C_{K} \in E_{*, K}} C_{K}, K=1$, $2, \ldots, N$, there exist vector functions $\bar{p}_{K}(t), K=1, \ldots, N$ for which the following necessary optimality condition holds:

(i) State equation:

$$
\dot{\bar{x}}_{K}(t)=\frac{\partial H_{K}\left(\bar{x}_{K}(t), \bar{u}_{K}(t), \bar{p}_{K}(t), t\right)}{\partial p_{K}}, \quad t \in\left[\bar{t}_{K-1}, \bar{t}_{K}\right] ;
$$

(ii) Costate equation:

$$
\dot{\bar{p}}_{K}(t)=\frac{\partial H_{K}\left(\bar{x}_{K}(t), \bar{u}_{K}(t), \bar{p}_{K}(t), t\right)}{\partial x_{K}}, \quad t \in\left[\bar{t}_{K-1}, \bar{t}_{K}\right] ;
$$

(iii) At the switching points, $\bar{t}_{1}, \bar{t}_{2}, \ldots, \bar{t}_{N-1}$,

$$
\begin{array}{r}
x_{K}^{*}-\bar{p}_{K}\left(\bar{t}_{K}\right)-\bar{p}_{K+1}\left(\bar{t}_{K}\right) \frac{\partial M_{K}\left(\bar{x}_{K}\left(\bar{t}_{K}\right), \bar{t}_{K}\right)}{\partial x_{K}}=0, \\
K=1,2, \ldots, N-1 ;
\end{array}
$$

(iv) Minimality condition:

$$
\begin{gathered}
H_{K}\left(\bar{x}_{K}(t), \bar{p}_{K}(t), \bar{u}_{K}(t)+\delta u_{K}(t), t\right) \\
\quad \geq H_{K}\left(\bar{x}_{K}(t), \bar{p}_{K}(t), \bar{u}_{K}(t), t\right), \\
\text { for all admissible } \delta u_{K}, \quad t \in\left[\bar{t}_{K-1}, \bar{t}_{K}\right] ;
\end{gathered}
$$

(v) At the end point $\bar{t}_{N}$,

$$
\bar{p}_{N}\left(\bar{t}_{N}\right)=x_{N}^{*}+\sum_{K=1}^{N} \lambda_{K} \frac{\partial F_{K}\left(\bar{x}_{N}\left(\bar{t}_{N}\right), \bar{t}_{N}\right)}{\partial x_{N}},
$$

$$
\begin{aligned}
\left(\sum_{K=1}^{N} \lambda_{K} \frac{\partial F_{K}\left(\bar{x}_{N}\left(\bar{t}_{N}\right), \bar{t}_{N}\right)}{\partial t_{N}}\right) \delta_{L, N} \\
-\frac{1}{N}\left(\sum_{K=1}^{N-1} \bar{p}_{K+1}\left(\bar{t}_{K}\right) \frac{\partial M_{K}\left(\bar{x}_{K}\left(\bar{t}_{K}\right), \bar{t}_{K}\right)}{\partial t_{K}}\right)\left(1-\delta_{L, N}\right)=0,
\end{aligned}
$$

here

$$
\delta_{L, N}=\left\{\begin{array}{ll}
1, & L=N, \\
0, & L \neq N,
\end{array} \quad L=1,2, \ldots, N\right.
$$

is a Kronecker symbol, $H_{K}\left(x_{K}, u_{K}, p_{K}, t\right)=L_{K}\left(x_{K}\right.$, $\left.u_{K}, p_{K}, t\right)+p_{K}^{T} \cdot f_{K}\left(x_{K}, u_{K}, p_{K}, t\right)$, is a HamiltonPontryagin function, $E_{*, K}$ is lower exhauster of the functional $\varphi_{K}\left(x_{K}\left(t_{K}\right)\right), \lambda_{K}, K=1, \ldots, N$ are the vectors, and $p_{k}(\cdot)$ is defined by the conditions (ii) and (iii) in the process of the proof of the theorem, later.

Proof. Firstly, we will try to reduce optimal control problem (14)-(18) with nonsmooth cost functional to the optimal control problem with smooth minimization functional. In this way, we will use some useful theorems in $[12,13]$. Let us note that smooth variational descriptions of Frechet normals theorem in [12, Theorem 1.30] and its subdifferential counterpart [12, Theorem 1.88] provide important variational descriptions of Frechet subgradients of nonsmooth functions in terms of smooth supports. To prove the theorem, take any elements from intersection of the subset of the exhauster, $x_{K}^{*} \in \bigcap C_{K}$, where $C_{K} \in E_{*, K}, K=1,2, \ldots, N$. Then by using Theorem 1, we can write that $x_{K}^{*} \in \widehat{\partial}^{+} \varphi_{K}\left(\bar{x}_{K}\left(t_{K}\right)\right)$. Then, apply the variational description in [12, Theorem 1.88] to the subgradients $-x_{K}^{*} \in \widehat{\partial}^{+}\left(-\varphi_{K}\left(\bar{x}_{K}\left(\bar{t}_{K}\right)\right)\right)$. In this way, we find functions $s_{K}$ : $X \rightarrow \mathbb{R}$ for $K=1,2, \ldots, N$ satisfying the relations

$$
s_{K}\left(\bar{x}_{K}\left(t_{K}\right)\right)=\varphi_{K}\left(\bar{x}_{K}\left(t_{K}\right)\right), \quad s_{K}\left(x_{K}(t)\right) \geq \varphi_{K}\left(x_{K}(t)\right)
$$

in some neighborhood of $\bar{x}_{K}\left(t_{K}\right)$, and such that each $s_{K}(\cdot)$ is continuously differentiable at $\bar{x}_{K}\left(\bar{t}_{K}\right)$ with $\nabla s_{K}\left(\bar{x}_{K}\left(s \bar{t}_{K}\right)\right)=$ $x_{K}^{*}, K=1,2, \ldots, N$. It is easy to check that $\bar{x}_{K}(\cdot)$ is a local solution to the following optimization problem of type (14)(18) but with cost continuously differentiable around $\bar{x}_{K}(\cdot)$. This means that we deduce the optimal control problem (14)(18) with the nonsmooth cost functional to the smooth cost functional data:

$$
\begin{aligned}
\min S & \left(u_{1}, \ldots, u_{N}, t_{1}, \ldots, t_{N}\right) \\
& =\sum_{K=1}^{N} s_{K}\left(x_{K}\left(t_{K}\right)\right)+\sum_{K=1}^{N} \int_{t_{K-1}}^{t_{K}} L\left(x_{K}(t), u_{K}(t), t\right) d t,
\end{aligned}
$$

taking into account that

$$
\nabla s_{K}\left(\bar{x}_{K}\left(\bar{t}_{K}\right)\right)=x_{K}^{*}, \quad K=1,2, \ldots, N .
$$


We use multipliers to adjoint to constraints $\dot{x}_{K}(t)-$ $f_{K}\left(x_{K}(t), u_{K}(t), t\right)=0, t \in\left[t_{k-1}, t_{k}\right], K=1,2, \ldots, N$ and $F_{K}\left(x_{N}\left(t_{N}\right), t_{N}\right)=0, K=1,2, \ldots, N$ to $S$ :

$$
\begin{aligned}
J^{\prime}= & \sum_{K=1}^{N} s_{K}\left(x_{K}\left(t_{K}\right)\right)+\sum_{K=1}^{N} \lambda_{k} F_{K}\left(x_{N}\left(t_{N}\right), t_{N}\right) \\
& +\sum_{K=1}^{N} \int_{t_{K-1}}^{t_{K}}\left(L\left(x_{K}(t), u_{K}(t), t\right)+p_{K}^{T}(t)\right. \\
& \left.\times\left(f_{K}\left(x_{K}(t), u_{K}(t), t\right)-\dot{x}_{K}(t)\right)\right) d t
\end{aligned}
$$

by introducing the Lagrange multipliers $p_{1}(t), p_{2}(t), \ldots$, $p_{N}(t)$. In the following, we will find it convenient to use the function $H_{K}$, called the Hamiltonian, defined as $H_{K}\left(x_{K}(t), p_{K}(t), u_{K}(t), t\right)=L_{K}\left(x_{K}(t), u_{K}(t), t\right)+$ $p_{K}(t) f_{K}\left(x_{K}, u_{K}, t\right)$ for $t \in\left[t_{K-1}, t_{K}\right]$. Using this notation, we can write the Lagrange functional as

$$
\begin{aligned}
J^{\prime}= & \sum_{K=1}^{N} s_{K}\left(x_{K}\left(t_{K}\right)\right)+\sum_{K=1}^{N} \lambda_{k} F_{K}\left(x_{N}\left(t_{N}\right), t_{N}\right) \\
& +\sum_{K=1}^{N} \int_{t_{K-1}}^{t_{K}}\left(H_{K}\left(x_{K}(t), p_{K}(t), u_{K}(t), t\right)-p_{K}^{T} \dot{x}_{K}(t)\right) d t .
\end{aligned}
$$

Assume $\left\{\bar{x}_{k}, \bar{u}_{k}, \bar{\theta}_{k}\right\}$ is optimal control. To determine the variation $\delta J^{\prime}$, we introduce the variation $\delta x_{K}, \delta u_{K}, \delta p_{K}$, and $\delta t_{K}$. From the calculus of variations, we can obtain that the first variation of $J^{\prime}$ as

$$
\begin{aligned}
\delta J^{\prime}= & \sum_{K=1}^{N} \frac{\partial s_{K}\left(\bar{x}_{K}\left(\bar{t}_{K}\right)\right)}{\partial x_{K}} \delta x_{K}\left(t_{K}\right) \\
& +\sum_{K=1}^{N} \lambda_{K} \frac{\partial F_{K}\left(\bar{x}_{N}\left(\bar{t}_{N}\right), \bar{t}_{N}\right)}{\partial x_{N}} \delta x_{N}\left(t_{N}\right) \\
& +\sum_{K=1}^{N} \lambda_{K} \frac{\partial F_{K}\left(\bar{x}_{N}\left(\bar{t}_{N}\right), \bar{t}_{K}\right)}{\partial t_{N}} \delta t_{N} \\
& +\sum_{K=1}^{N} \int_{t_{K-1}}^{t_{K}} \frac{\partial H_{K}\left(\bar{x}_{K}(t), \bar{u}_{K}(t), \bar{p}_{K}(t), t\right)}{\partial x_{K}} \delta x_{K}(t) \\
& +\sum_{K=1}^{N} \int_{t_{K-1}}^{t_{K}} \frac{\partial H_{K}\left(\bar{x}_{K}(t), \bar{u}_{K}(t), \bar{p}_{K}(t), t\right)}{\partial u_{K}} \delta u_{K} \\
& +\sum_{K=1}^{N} \int_{t_{K-1}}^{t_{K}} \frac{\partial H_{K}\left(\bar{x}_{K}(t), \bar{u}_{K}(t), \bar{p}_{K}(t), t\right)}{\partial p_{K}} \delta p_{K} \\
& -\sum_{K=1}^{N} \int_{t_{K-1}}^{t_{K}}\left(\bar{p}_{K}(t) \delta x_{K}(t)+\bar{x}_{K}(t) \delta p_{K}(t)\right) d t
\end{aligned}
$$

+ high order terms.
If we follow the steps in [3, pages 5-7] then, the first variation of the functional takes the following form:

$$
\begin{aligned}
& \delta J^{\prime}=\sum_{K=1}^{N-1} \frac{\partial s_{K}\left(\bar{x}_{K}\left(\bar{t}_{K}\right)\right)}{\partial x_{K}} \delta x_{K}\left(t_{K}\right)+\frac{\partial s\left(\bar{x}_{N}\left(\bar{t}_{N}\right)\right)}{\partial x_{N}} \delta x_{N}\left(t_{N}\right) \\
& +\sum_{K=1}^{N} \lambda_{K} \frac{\partial F_{K}\left(\bar{x}_{N}\left(\bar{t}_{N}\right), \bar{t}_{N}\right)}{\partial x_{N}} \delta x_{N}\left(t_{N}\right) \\
& +\sum_{K=1}^{N} \lambda_{K} \frac{\partial F_{K}\left(\bar{x}_{N}\left(\bar{t}_{N}\right), \bar{t}_{N}\right)}{\partial t_{N}} \delta t_{N} \\
& +\sum_{K=1}^{N} \int_{t_{K-1}}^{t_{K}} \frac{\partial H_{K}\left(\bar{u}_{K}, \bar{x}_{K}, \bar{p}_{K}, t\right)}{\partial u_{K}} \delta u_{K} \\
& +\sum_{K=1}^{N} \int_{t_{K-1}}^{t_{K}} \frac{\partial H_{K}\left(\bar{u}_{K}(t), \bar{x}_{K}(t), \bar{p}_{K}(t), t\right)}{\partial \bar{p}_{K}} \delta p_{K} \\
& -\sum_{K=1}^{N-1} \bar{p}_{K}\left(t_{K}\right) \delta x_{K}\left(t_{K}\right)-\bar{p}_{N}\left(t_{N}\right) \delta x_{N}\left(t_{N}\right) \\
& -\sum_{K=1}^{N-1} p_{K+1}\left(\bar{t}_{K}\right) \frac{\partial M_{K}\left(\bar{x}_{K}\left(\bar{t}_{K}\right), \bar{t}_{K}\right)}{\partial x_{K}} \delta x_{K}\left(\bar{t}_{K}\right) \\
& -\sum_{K=1}^{N-1} \bar{p}_{K+1}\left(\bar{t}_{K}\right) \frac{\partial M_{K}\left(\bar{x}_{K}\left(\bar{t}_{K}\right), \bar{t}_{K}\right)}{\partial t_{K}} \delta t_{K} \\
& -\sum_{k=1}^{N} \bar{p}_{K}(t) \delta x_{K}\left(t_{K}\right) \\
& =\sum_{K=1}^{N-1}\left(\frac{\partial \varphi_{K}\left(\bar{x}_{K}\left(\bar{t}_{K}\right)\right)}{\partial x_{K}}-\bar{p}_{K}\left(\bar{t}_{K}\right)\right. \\
& \left.-\bar{p}_{K+1}\left(\bar{t}_{K}\right) \frac{\partial M_{K}\left(\bar{x}_{K}\left(\bar{t}_{K}\right), \bar{t}_{K}\right)}{\partial x_{K}}\right) \delta x_{K}\left(\bar{t}_{K}\right) \\
& +\left(\frac{\partial \varphi_{N}\left(\bar{x}_{N}\left(\bar{t}_{N}\right)\right)}{\partial x_{N}}+\sum_{K=1}^{N} \lambda_{K} \frac{\partial F_{K}\left(\bar{x}_{N}\left(\bar{t}_{N}\right), \bar{t}_{N}\right)}{\partial \bar{x}_{N}}\right. \\
& \left.-\bar{p}_{N}\left(t_{N}\right)\right) \delta x_{N}\left(t_{N}\right) \\
& +\sum_{L=1}^{N}\left[\left(\sum_{K=1}^{N} \lambda_{K} \frac{\partial F_{K}\left(\bar{x}_{N}\left(\bar{t}_{N}\right), \bar{t}_{N}\right)}{\partial \bar{t}_{N}}\right) \delta_{L, N}\right. \\
& -\frac{1}{N}\left(\sum_{K=1}^{N-1} \bar{p}_{K+1}\left(\bar{t}_{K}\right) \frac{\partial M_{K}\left(\bar{x}_{K}\left(\bar{t}_{K}\right), \bar{t}_{K}\right)}{\partial t_{K}}\right) \\
& \left.\times\left(1-\delta_{L, N}\right)\right] \delta t_{L} \\
& +\sum_{K=1}^{N} \int_{\bar{t}_{K-1}}^{\bar{t}_{K}}\left(\frac{\partial H_{K}\left(\bar{x}_{K}(t), \bar{p}_{K}(t), \bar{u}_{K}(t), t\right)}{\partial x_{K}}-\dot{\bar{p}}_{K}(t)\right) \delta x_{K} \\
& +\sum_{K=1}^{N} \int_{\bar{t}_{K-1}}^{\bar{t}_{K}} \frac{\partial H_{K}\left(\bar{x}_{K}(t), \bar{p}_{K}(t), \bar{u}_{K}(t), t\right)}{\partial u_{K}} \delta u_{K} \\
& +\sum_{K=1}^{N} \int_{t_{K-1}}^{t_{K}}\left(\frac{\partial H_{K}\left(\bar{x}_{K}(t), \bar{p}_{K}(t), \bar{u}_{K}(t), t\right)}{\partial p_{K}}-\dot{\bar{x}}_{K}(t)\right) \delta p_{K} .
\end{aligned}
$$


The latter sum is known because

$$
\frac{\partial H_{K}\left(\bar{x}_{K}(t), \bar{u}_{K}(t), \bar{p}_{K}(t), t\right)}{\partial p_{K}}=\dot{\bar{x}}_{K}(t),
$$

and it is easy to check that

$$
\begin{gathered}
\sum_{K=1}^{N} \lambda_{K} \frac{\partial F_{K}\left(\bar{x}_{N}\left(\bar{t}_{N}\right), \bar{t}_{N}\right)}{\partial t_{N}} \delta t_{N} \\
-\sum_{K=1}^{N-1} \bar{p}_{K+1}\left(\bar{t}_{K}\right) \frac{\partial M_{K}\left(\bar{x}_{K}\left(\bar{t}_{K}\right), \bar{t}_{K}\right)}{\partial t_{K}} \delta t_{K} \\
=\sum_{L=1}^{N}\left[\left(\sum_{K=1}^{N} \lambda_{K} \frac{\partial F_{K}\left(\bar{x}_{N}\left(\bar{t}_{N}\right), \bar{t}_{N}\right)}{\partial t_{N}}\right) \delta_{L, N}{ }^{N}\right. \\
\quad-\frac{1}{N}\left(\sum_{K=1}^{N-1} \bar{p}_{K+1}\left(\bar{t}_{K}\right) \frac{\partial M_{K}\left(\bar{x}_{K}\left(\bar{t}_{K}\right), \bar{t}_{K}\right)}{\partial t_{K}}\right) \\
\left.\times\left(1-\delta_{L, N}\right)\right] \delta t_{L} .
\end{gathered}
$$

If the state equations (14) are satisfied, $\dot{p}_{k}$ is selected so that coefficient of $\delta x_{k}$ and $\delta t_{N}$ is identically zero. Thus, we have

$$
\begin{aligned}
\delta S^{\prime}= & \sum_{K=1}^{N-1} \int_{t_{K-1}}^{t_{K}} \frac{\partial H_{K}\left(\bar{x}_{K}(t), \bar{p}_{K}(t), \bar{u}_{K}(t), t\right)}{\partial u_{K}} \delta u_{K} \\
& + \text { high order terms. }
\end{aligned}
$$

The integrand is the first-order approximation to the change in $H_{K}$ caused by

$$
\begin{gathered}
{\left[\frac{\partial H_{K}\left(\bar{x}_{K}(t), \bar{p}_{K}(t), \bar{u}_{K}(t), t\right)}{\partial u_{K}} \delta u_{K}\right]^{T} \delta u_{K}(t)} \\
=H_{K}\left(\bar{x}_{K}(t), \bar{p}_{K}(t), \bar{u}_{K}(t)+\delta u_{K}(t), t\right) \\
\quad-H_{K}\left(\bar{x}_{K}(t), \bar{p}_{K}(t) \bar{u}_{K}(t), t\right) .
\end{gathered}
$$

Therefore,

$$
\begin{aligned}
\delta S^{\prime}=\sum_{K=1}^{N-1} \int_{t_{K-1}}^{t_{K}}[ & H_{K}\left(\bar{x}_{K}(t), \bar{p}_{K}(t), \bar{u}_{K}(t)+\delta u_{K}(t), t\right) \\
& \left.-H_{K}\left(\bar{x}_{K}(t), \bar{p}_{K}(t), \bar{u}_{K}(t), t\right)\right] \delta u_{K}(t)
\end{aligned}
$$

+ high order terms.

If $\bar{u}_{K}+\delta u_{K}$ is in a sufficiently small neighborhood of $\bar{u}_{K}$ then the high-order terms are small and the integral in last equation dominates the expression of $\delta S^{\prime}$. Thus, for $\bar{u}_{K}$ to be a minimizing control it is necessary that

$$
\begin{aligned}
\sum_{K=1}^{N-1} \int_{t_{K-1}}^{t_{K}}\left[H_{K}\left(\bar{x}_{K}, \bar{p}_{K}, \bar{u}_{K}+\delta u_{K}, t\right)\right. \\
\left.\quad-H_{K}\left(\bar{x}_{K}, \bar{p}_{K}, \bar{u}_{K}, t\right)\right] \delta u_{K} \geq 0
\end{aligned}
$$

for all admissible $\delta u_{K}$. We assert that in order for the last inequality to be satisfied for all admissible $\delta u_{K}$ in the specified neighborhood, it is necessary that $H_{K}\left(\bar{x}_{K}, \bar{p}_{K}, \bar{u}_{K}+\delta u_{K}, t\right) \geq$ $H_{K}\left(\bar{x}_{K}, \bar{p}_{K}, \bar{u}_{K}, t\right)$ for all admissible $\delta u_{K}$ and for all $t \in$ $\left[t_{K-1}, t_{K}\right]$. To show this, consider the control

$$
\Delta u_{K}= \begin{cases}\bar{u}_{K}(t), & t \in\left[t_{K-1}, t_{K}\right], \\ \bar{u}_{K}(t)+\delta u_{K}(t), & t \in\left[t_{K-1}, t_{K}\right],\end{cases}
$$

where $t \in\left[t_{K-1}, t_{K}\right]$ is an arbitrarily small, but nonzero time interval and $\delta u_{K}$ are admissible control variations. After this, if we consider proof description of the maximum principle in [4], we can come to the last inequality.

According to the fundamental theorem of the calculus of the variation, at the extremal point the first variation of the functional must be zero, that is, $\delta J^{\prime}=0$. Setting to zero, the coefficients of the independent increments $\delta x_{N}\left(t_{N}\right)$, $\delta x_{K}\left(t_{K}\right) \delta x_{K}, \delta u_{K}$ and $\delta p_{K}$, and taking into account that

$$
\nabla s_{K}\left(\bar{x}_{K}\left(t_{K}\right)\right)=x_{K}^{*}, \quad K=1,2, \ldots, N,
$$

yield the necessary optimality conditions (i)-(v) in Theorem 3.

This completes the proof of the theorem.

Theorem 4 (Necessary optimality conditions for switching optimal control system in terms of Quasidiffereniability). Let the minimization functional $\varphi_{K}(\cdot)$ be positively homogenous, quasidifferentiable at a point $\bar{x}_{K}(\cdot)$, and let $\left(\bar{u}_{K}(\cdot), \bar{x}_{K}(\cdot), \bar{\theta}\right)$ be an optimal solution to the control problem (14)-(18). Then, there exist vector functions $p_{K}(t), K=1, \ldots, N$, and there exist convex compact and bounded set $M\left(\varphi_{K}(\cdot)\right)$, in which for any elements $x_{K}^{*} \in M\left(\varphi_{K}(\cdot)\right)$, the necessary optimality conditions (i)-(v) in Theorem 3 are satisfied.

Proof. Let minimization functional $\varphi_{K}(\cdot)$ be positively homogenous and quasidifferentiable at a point $\bar{x}_{K}\left(\bar{t}_{K}\right)$. Then, there exist totally bounded lower exhausters $E_{*, K}$ for the $\varphi_{K}(\cdot)$ [9, Theorem 4]. Let us make the substitution $M\left(\varphi_{K}(\cdot)\right)=$ $E_{*, K}$; take any element $x_{K}^{*} \in M\left(\varphi_{K}(\cdot)\right)$, then $x_{K}^{*} \in E_{*, K}$ also, and if we follow the proof description and result in Theorem 3 in the current paper, we can prove Theorem 4 . If we use the relationship between the Gateaux upper subdifferential and Dini upper derivative [9, Lemma 3.6], substitute $h_{K}(g)=$ $\varphi_{K, H}^{+}\left(\bar{x}_{K}\left(\bar{t}_{K}\right), g\right)$, then we can write the following corollary (here $\varphi_{K, H}^{+}\left(\bar{x}_{K}\left(\bar{t}_{K}\right), g\right.$ is the Hadamard upper derivative of the minimizing functional $\varphi_{K}(\cdot)$ in the direction $g$ ).

Corollary 5. Let the minimization functional $\varphi_{K}(\cdot)$ be positively homogenous, and let the Dini upper differentiable at a point $x_{K}\left(\bar{t}_{K}\right)$ and $\left(\bar{u}_{K}(\cdot), \bar{x}_{K}(\cdot), \bar{\theta}\right)$ be an optimal solution to the control problem (14)-(18). Then for any elements $x_{K}^{*} \in$ $\partial_{G}^{+} h_{K}\left(0_{n}\right)$, there exist vector functions $p_{K}(t), K=1, \ldots, N$ in which the necessary optimality conditions $(i)-(v)$ in the Theorem 3 hold.

Proof. Let us take any element $x_{K}^{*} \in \partial_{G}^{+} h_{K}\left(0_{n}\right)$. Then by using the lemma in [9, Lemma 3.8] we can write $x_{K}^{*} \in \partial_{F}^{+} h_{K}\left(0_{n}\right)$. Next, if we use the lemma in [9, Lemma 3.2], then we can put 
$x_{K}^{*} \in \partial_{F}^{+} \varphi_{K}\left(\bar{x}_{K}\left(\bar{t}_{K}\right)\right)$. At least, if we follow Theorem 1 (relationship between upper Frechet subdifferential and exhausters) and Theorem 3 (necessary optimality condition in terms of exhausters) in the current paper, we can prove the result of Corollary 5.

\section{References}

[1] P. J. Antsaklis and A. Nerode, "Special issue on hybrid system," IEEE Transactions on Automatic Control, vol. 43, no. 4, pp. 540$554,1998$.

[2] A. Bensoussan and J. L. Menaldi, "Hybrid control and dynamic programming," Dynamics of Continuous, Discrete and Impulsive Systems, vol. 3, no. 4, pp. 395-442, 1997.

[3] S. F. Maharramov, "Necessary optimality conditions for switching control problems," Journal of Industrial and Management Optimization, vol. 6, no. 1, pp. 47-55, 2010.

[4] V. Boltyanski, "The maximum principle for variable structure systems," International Journal of Control, vol. 77, no. 17, pp. 1445-1451, 2004.

[5] S. F. Magerramov and K. B. Mansimov, "Optimization of a class of discrete step control systems," Zhurnal Vychislitel'noi Matematiki i Matematicheskoi Fiziki, vol. 41, no. 3, pp. 360-366, 2001.

[6] R. M. Caravello and B. Piccoli, "Hybrid necessary principle," in Proceedings of the 44th IEEE Conference on Decision and Control, pp. 723-728, 2002.

[7] S. F. Maharramov, "Optimality condition of a nonsmooth switching control system," Automotic Control and Computer Science, vol. 42, no. 2, pp. 94-101, 2008.

[8] V. F. Demyanov and V. A. Roshchina, "Constrained optimality conditions in terms of proper and adjoint exhausters," Applied and Computational Mathematics, vol. 4, no. 2, pp. 114-124, 2005.

[9] V. F. Demyanov and V. Roshchina, "Exhausters and subdifferentials in non-smooth analysis," Optimization, vol. 57, no. 1, pp. 41-56, 2008.

[10] V. F. Demyanov and V. A. Roshchina, "Exhausters, optimality conditions and related problems," Journal of Global Optimization, vol. 40, no. 1-3, pp. 71-85, 2008.

[11] F. Tröltzsch, Optimal Control of Partial Differential Equations, Theory, Methods and Applications, American Mathematical Society, 2010.

[12] B. S. Mordukhovich, Variational Analysis and Generalized Differentiation I: Basic Theory, Springer, Berlin, Germany, 2006.

[13] B. S. Mordukhovich, Variational Analysis and Generalized Differentiation, II: Applications, Springer, Berlin, Germany, 2006. 


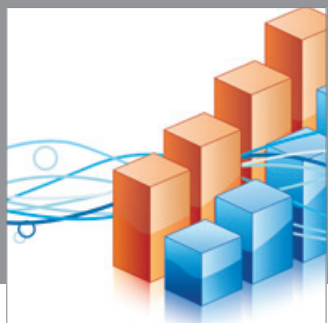

Advances in

Operations Research

mansans

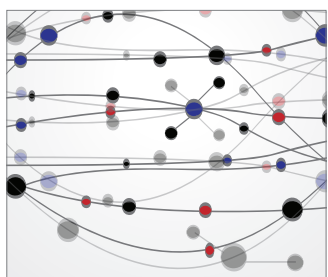

The Scientific World Journal
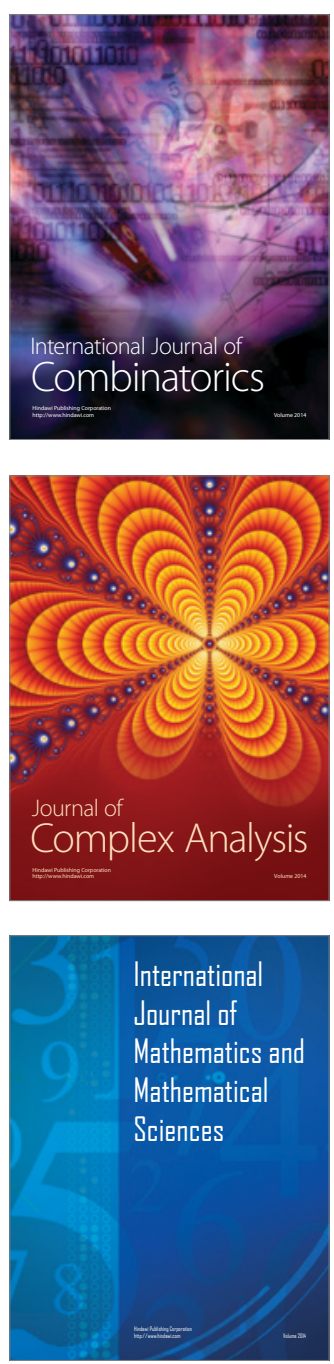
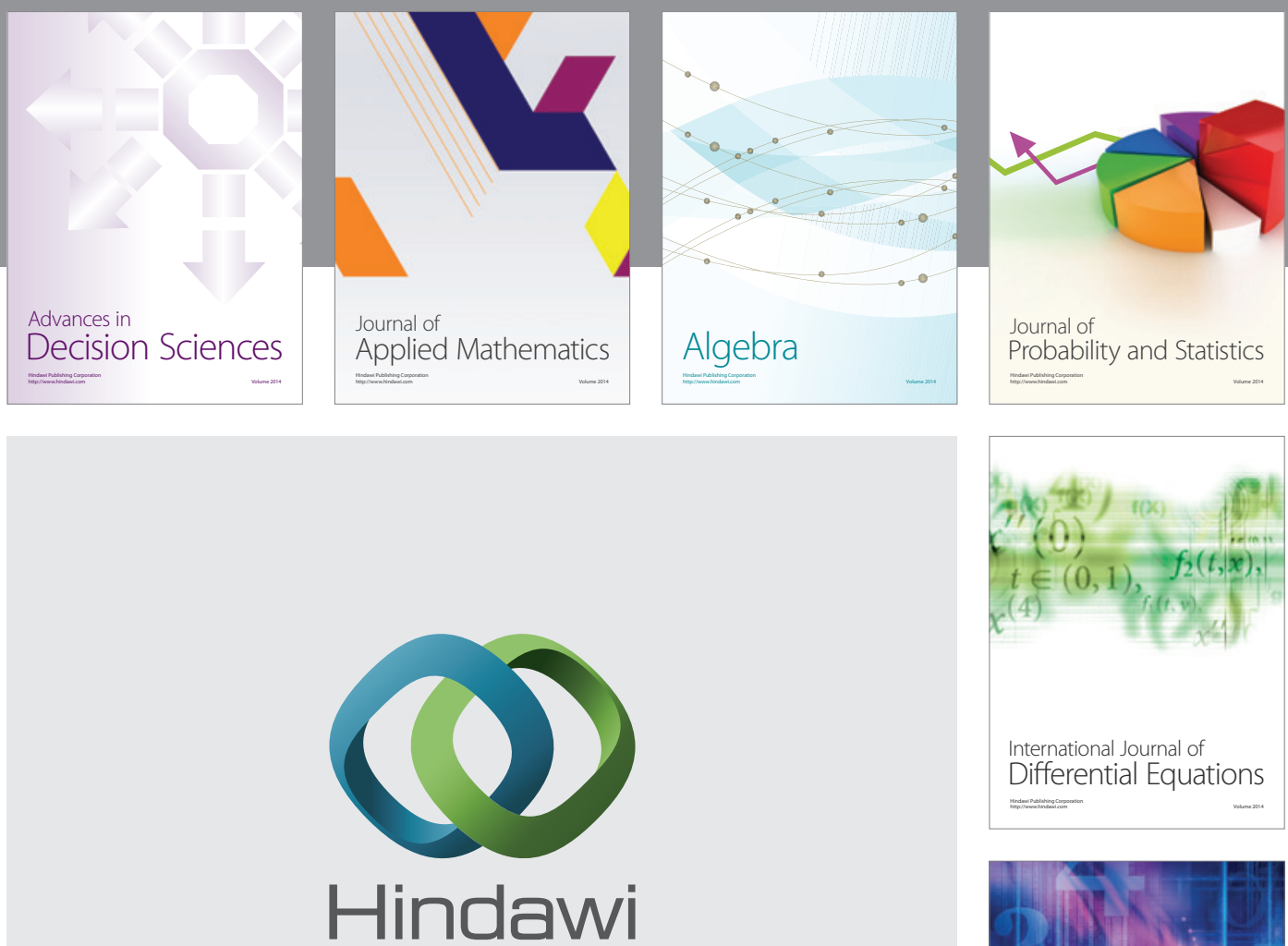

Submit your manuscripts at http://www.hindawi.com
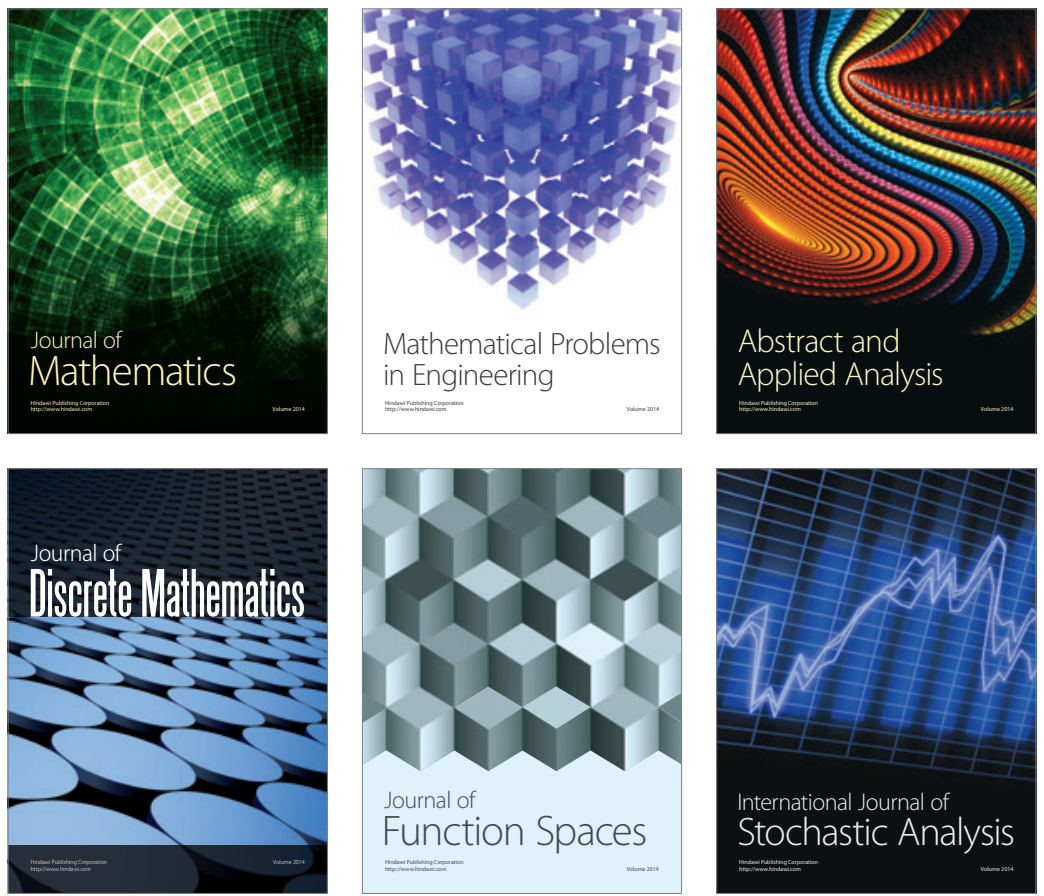

Journal of

Function Spaces

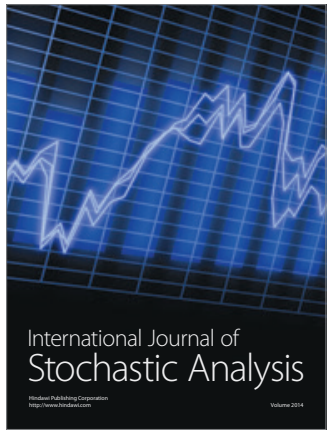

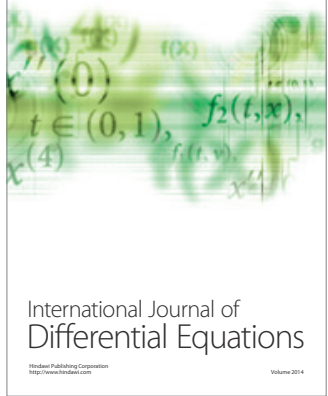
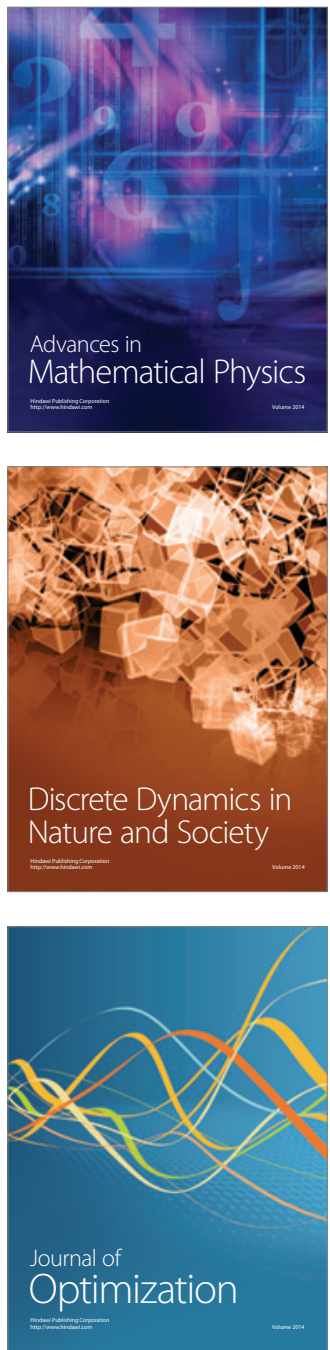